lung und Umsetzung einer Markenstrategie gegliedert. Dieses Praxishandbuch ist differenziert, anspruchsvoll und gelungen und kann der Praxis wertvolle Impulse geben. Es löst seinen Anspruch vorzüglich ein.

Cornelia Briel: Die Bücherlager der Reichstauschstelle. Mit einem Vorwort von Georg Ruppelt. Frankfurt am Main: Klostermann, 2016. (Zeitschrift für Bibliothekswesen und Bibliographie; Sonderband 117). 360 S., 38 s/w. Abb., fest geb. ISBN 978-3-465-04249-5, ISSN 0514-6364.€ 94,-

Besprochen von Prof. Dr. Peter Vodosek: Seestraße 89, D-70174 Stuttgart, E-Mail: vodosek@hdm-stuttgart.de

\section{DOI 10.1515/bfp-2017-0023}

Cornelia Briel, gegenwärtig mit dem Projekt Publikationsstelle Berlin-Dahlem in der Forschungsbibliothek des Herder-Instituts beschäftigt, ist als Provenienzforscherin bestens ausgewiesen. Das Literaturverzeichnis dieser Neuerscheinung führt aus den Jahren zwischen 2007 und 2014 fünf einschlägige Arbeiten von ihr an, von denen drei die Reichstauschstelle zum Gegenstand haben. 2013 legte sie die erste umfassende Studie über diese Dienststelle vor. ${ }^{1}$ Georg Ruppelt würdigt im Vorwort des vorliegenden Buches ausführlich diese vorhergehende Arbeit. Er gibt einen Überblick über den schwer zu entwirrenden Zusammenhang zwischen der 1926 gegründeten Reichstauschstelle, dem Beschaffungsamt der Deutschen Bibliotheken und dem Deutsch-Ausländischen Buchaustausch, die 1941 zusammengefasst und mit der Preußischen Staatsbibliothek verbunden wurden. Detaillierter kommt die Autorin im zweiten Kapitel darauf zu sprechen (S. 31-33).

Hatte die erste Studie von 2013, die aus einem von 2006 bis 2009 laufenden Projekt hervorgegangen war, die Aktivitäten und das Umfeld der Reichstauschstelle im Fokus, beschäftigt sich diese zweite schwerpunktmäßig mit den Bücherlagern und Depots, welche die Stelle weiträumig gestreut anlegte. Im November 1944 verfügte die Reichstauschstelle über 33 Depots (S. 155, Anm. 266). Um Missverständnisse zu vermeiden: Hier geht es nicht um die

1 Briel, Cornelia (2013): Beschlagnahmt, erpresst, erbeutet: NS-Raubgut, Reichstauschstelle und Preußische Staatsbibliothek zwischen 1933 und 1945, hrsg.v. Hans Erich Bödeker und Gerd-Josef Bötte in Zusammenarbeit mit der Staatsbibliothek Berlin - Preußischer Kulturbesitz. Mit einem Geleitwort von Barbara Schneider-Kempf. Berlin: Akademie-Verlag. Vgl. dazu Rezension von Juliane Deinert (2014) in BIBLIOTHEK - Forschung und Praxis 38 (3), 513-516.
Verlagerungsaktionen als Rettungsoperationen für die Preußische Staatsbibliothek und andere Bibliotheken im Reich, sondern um die Einlagerung der von der Reichsstelle für den Wiederaufbau erworbenen Literatur nach kriegsbedingten Zerstörungen. ${ }^{2}$

Es kann nicht Aufgabe einer Rezension sein, Briels Studie zu einer Kurzfassung zu komprimieren. Zum besseren Verständnis aber sollen in aller Kürze die wichtigsten Fakten referiert werden, wie sie sich auch in der Einleitung (S. 13-30) finden. Zunächst ist festzuhalten, dass „die Vorgänge um die Bücherlager der Reichstauschstelle und damit die Herkunft und der Verbleib von mehreren Hunderttausend Bänden ,Wiederaufbauliteratur' [...] in der Forschungsliteratur bislang kaum thematisiert [wurde]“. Diese Feststellung der Autorin zu zitieren, lässt bereits die Herausforderung ahnen, mit der sie sich konfrontiert sah. 1943 beauftragte das Reichsministerium für Wissenschaft, Erziehung und Volksbildung die Reichstauschstelle mit dem Wiederaufbau der bei den Luftangriffen zerstörten oder beschädigten deutschen wissenschaftlichen Bibliotheken. Der Reichsminister der Finanzen stellte dafür fünf Millionen Reichsmark zur Verfügung. Briel untersucht nun auf der Grundlage der Aktenüberlieferung Verteilung, Einlagerung und Verbleib der beschafften Literatur. Sie schätzt, dass zwischen einem Drittel und einem Viertel die „Wiederaufbauliteratur“ aus beschlagnahmten und eingezogenen Beständen, also aus NS-Raubgut, bestand, die sie von anderen Dienststellen ankaufte. Sie tätigte darüber hinaus legitime und quasilegale Ankäufe im Buchhandel und von Privatpersonen, die kritisch hinterfragt werden müssen. ${ }^{3}$ Darüber hinaus war die Anlage von Depots für amtliche Druckschriften ein wichtiger Gesichtspunkt, um nach Zerstörungen im Rahmen des Wiederaufbaues eine geordnete Verwaltung weiterführen zu können. Schätzungen nach Kriegsende zufolge belief sich die Gesamtzahl der eingelagerten Literatur um die 900000 Bände.

2 Die Verlagerungsgeschichte der Preußischen Staatsbibliothek wurde von Werner Schochow (2003) erforscht: Bücherschicksale. Die Verlagerungsgeschichte der Preußischen Staatsbibliothek. Auslagerung Zerstörung - Entfremdung - Rückführung. Dargestellt aus den Quellen (Veröffentlichungen der Historischen Kommission zu Berlin; Bd. 102). Berlin, New York: Walter de Gruyter. Vgl. dazu die Rezension von Manfred Komorowski (2005) in BIBLIOTHEK - Forschung und Praxis 29 (2), 261-262.

3 Für Frankreich ergänzend hinzuziehen: Poulain, Martine (2008): Livres pillés, lectures surveillées. Les bibliothèques françaises sous l'Occupation (nfr Essais). Paris: Gallimard. Vgl. dazu die Rezension von Peter Vodosek (2009) in BIBLIOTHEK - Forschung und Praxis 33 (3), 405-407. 
Die zehn Kapitel der Studie lassen sich thematisch in fünf größeren Blöcken zusammenfassen:

- Das Zustandekommen des Wiederaufbauprogrammes,

- Das Verhältnis der Reichstauschstelle zu den geschädigten Bibliotheken,

- Anmietung, Belegung und Räumung der Depots,

- Abtransport und Verbleib der eingelagerten Bestände,

- Diskussion der Mengenangaben in den Depotverzeichnissen.

Erst ein Blick in das detaillierte Inhaltsverzeichnis zeigt den ganzen Umfang der erforderlichen Nachforschungen. So wurden die Bestände der Depots, viele von ihnen in Schlössern, Klöstern usw. angelegt, in Hessen, Thüringen, in der Lausitz, in Pommern, in der Uckermark, in der Mark Brandenburg und in Franken untersucht. Ihre Spuren werden nicht nur bis zu ihrer Auflösung verfolgt, sondern auch der Abtransport in die Sowjetische Besatzungszone und in die Westzonen. Ein Abschnitt beschäftigt sich auch mit dem Ende der Reichstauschstelle (S. 183-190). Ihr Leiter, Adolf Jürgens, starb Anfang November 1945 in amerikanischer Gefangenschaft. Gisela von Busse, seine Stellvertreterin, führte die Geschäfte der „Reichstauschstelle und des Beschaffungsamts der Deutschen Bibliotheken in Abwicklung“ ab November. In einem Exkurs (S. 142-146) wird auch die Systematik (Aktenzeichen und Signaturen) der Einlagerungen erläutert.
Abgerundet wird die Publikation durch eine Karte der in den Akten erwähnten, von der Reichstauschstelle genutzten oder geplanten Verlagerungsorte, die von Ostpreußen bis nach Lothringen, von Schleswig bis nach Salzburg über das damalige Reichsgebiet verteilt waren. Sogar Turin und Mailand finden sich auf der Karte. Unter den Abbildungen finden sich historische Fotos einiger Schlösser aus Privatbesitz, in denen Depots angelegt waren. Wichtiger noch sind die 34 Kopien von Aktennotizen, Berichten, Schriftwechseln, Einlagerungsplänen usw. Sie illustrieren nicht nur das Verwaltungshandeln, sondern ihnen sind wichtige zusätzliche Information zu entnehmen. Abschließend sei noch auf ein Abkürzungsverzeichnis, ein Verzeichnis der ungedruckten Quellen und ein umfängliches Literaturverzeichnis von 11 Seiten sowie ein Sachregister hingewiesen.

Wie schon die erste Studie von 2013 ist auch diese Dokumentation akribisch recherchiert. Sie ist darüber hinaus trotz des schwierigen Themas gut lesbar. Bei allen noch notwendigen weiteren Forschungen zum Problem von Provenienz und Restitution ist der immer wieder (nicht ganz zu Recht) beklagte Abstand vom bisher Geleisteten zum Wünschenswerten von Cornelia Briel signifikant verringert worden. 